\title{
Quality Indicator for Gastric Cancer Detection Based on Helicobacter pylori Status
}

\author{
Jae Myung Park ${ }^{1,2}$ \\ ${ }^{1}$ Division of Gastroenterology and Hepatology, Department of Internal Medicine, Seoul St. Mary's Hospital, College of Medicine, \\ ${ }^{2}$ Catholic Photomedicine Research Institute, The Catholic University of Korea, Seoul, Korea
}

See "Quality Indicators for the Detection of Helicobacter pylori-Negative Early Gastric Cancer: A Retrospective Observational Study" by Fumiaki Ishibashi, Konomi Kobayashi, Keita Fukushima, et al., on page 698-704.

Esophagogastroduodenoscopy (EGD) has been used to diagnose a wide variety of upper gastrointestinal (GI) disorders, predominantly upper GI cancers. Gastric cancer is the fifth most common malignancy and the third leading cause of cancer-related deaths worldwide. ${ }^{1}$ The high mortality rate due to gastric cancer is owing to late diagnosis. Late detection causes a 5 -year survival rate of approximately $24 \%$, which can be increased to $40 \%$, with early detection by means of screening. ${ }^{1,2}$ The purpose of gastric cancer screening is for early intestinal-type gastric cancer detection; this cancer type comprises more than $95 \%$ of all gastric cancers and has sequential stages from atrophy, through dysplasia, to cancer. ${ }^{3}$

Gastric cancer screening has been performed in high-incidence regions. Screening using EGD helps in the early detection of gastric cancer, thereby enabling the use of minimally invasive treatment modalities like endoscopic resection. However, quality indicators measuring endoscopy performance should be investigated because of the increasing need for quality control. Few studies have investigated whether the EGD

Received: October 13, 2020 Revised: November 16, 2020

Accepted: November 16, 2020

Correspondence: Jae Myung Park

Division of Gastroenterology and Hepatology, Department of Internal Medicine, Seoul St. Mary's Hospital, College of Medicine, The Catholic University of Korea, 222 Banpo-daero, Seocho-gu, Seoul 06591, Korea

Tel: +82-2-2258-6022, Fax: +82-2-2258-2089, E-mail: parkjerry@catholic.ac.kr ORCID: https://orcid.org/0000-0002-1534-7467

cc This is an Open Access article distributed under the terms of the Creative Commons Attribution Non-Commercial License (http://creativecommons.org/ licenses/by-nc/3.0) which permits unrestricted non-commercial use, distribution, and reproduction in any medium, provided the original work is properly cited. observation time is associated with the neoplasm detection rate. $^{4,5}$

Several previous studies reported a positive association between neoplasm detection rates and EGD observation time. A previous prospective study evaluated the association between EGD observation times and the detection rates of atrophic gastritis, intestinal metaplasia, and gastric tumors in 837 symptomatic patients ${ }^{5}$ and reported that endoscopists who observed lesions for over 7 minutes without biopsy showed a higher detection rate of high-risk lesions (HRLs) for gastric cancer. However, there were only 11 and 8 patients, respectively, with gastric cancers and gastric adenomas, which limited the accurate interpretation of the results. Furthermore, endoscopy trainees also performed EGD in this study. A Korean study retrospectively analyzed data of more than 100,000 patients who underwent screening EGD. ${ }^{4}$ In this study, the observation time was defined as the time to the end of observation after endoscope insertion into the duodenum. Endoscopists who observed lesions for more than 3 minutes without biopsy had a significantly higher neoplasm detection rate than those who observed lesions for less than 3 minutes. Subsequently, all endoscopists were asked to observe lesions for at least 3 minutes as an institutional policy, and the ensuing neoplasm detection rate was prospectively assessed. ${ }^{6}$ Consequently, endoscopists showed a significantly higher neoplasm detection rate. ${ }^{6}$ These previous studies consistently showed that observation time is a potentially important quality indicator of gastric cancer detection using EGD examination.

In this issue of Clinical Endoscopy, Ishibashi et al. ${ }^{7}$ reported 
the results of a retrospective analysis, i.e., the association between upper GI endoscopy examination time and the detection of HRLs including adenomas, carcinomas, or lymphomas in Japan between April 2017 and March 2019. During this period, 9 physicians performed EGD for 13,477 consecutive asymptomatic individuals. The average observation time for screening Helicobacter pylori (HP)-eradicated and HP-naïve patients was shorter than that for screening HP-positive patients. Multiple regression analyses showed that the observation time was an independent predictor for detecting HRLs in HP-eradicated patients. The authors concluded that observation time and endoscopist experience were quality indicators for detecting HRLs in HP-eradicated and HP-naïve patients, respectively.

Study limitations discussed by the authors were the inaccurate measurement of observation time, younger age of the study population relative to the general Japanese population, and small numbers of HRLs. Moreover, this study also had limitations such as the use of unclear study methods, lack of appropriate statistical analysis, and small number of participating endoscopists. The current study suggests that observation time and endoscopist experience may be determinants of endoscopic quality for EGD.
Conflicts of Interest

The author has no financial conflicts of interest.

\section{REFERENCES}

1. Torre LA, Bray F, Siegel RL, Ferlay J, Lortet-Tieulent J, Jemal A. Global cancer statistics, 2012. CA Cancer J Clin 2015;65:87-108.

2. Zhang X, Li M, Chen S, et al. Endoscopic screening in Asian countries is associated with reduced gastric cancer mortality: a meta-analysis and systematic review. Gastroenterology 2018;155:347-354.e9.

3. Correa P. A human model of gastric carcinogenesis. Cancer Res 1988;48:3554-3560

4. Park JM, Huo SM, Lee HH, Lee BI, Song HJ, Choi MG. Longer observation time increases proportion of neoplasms detected by esophagogastroduodenoscopy. Gastroenterology 2017;153:460-469.e1.

5. Teh JL, Tan JR, Lau LJ, et al. Longer examination time improves detection of gastric cancer during diagnostic upper gastrointestinal endoscopy. Clin Gastroenterol Hepatol 2015;13:480-487.e2.

6. Park JM, Kim SY, Shin G, et al. Implementation effect of institutional policy of esophagogastroduodenoscopy observation time on neoplasm detection. Gastrointest Endosc 2020 Sep 8 [Epub]. https://doi. org/10.1016/j.gie.2020.09.002.

7. Ishibashi F, Kobayashi K, Fukushima K, et al. Quality indicators for the detection of Helicobacter pylori-negative early gastric cancer: a retrospective observational study. Clin Endosc 2020;53:698-704. 